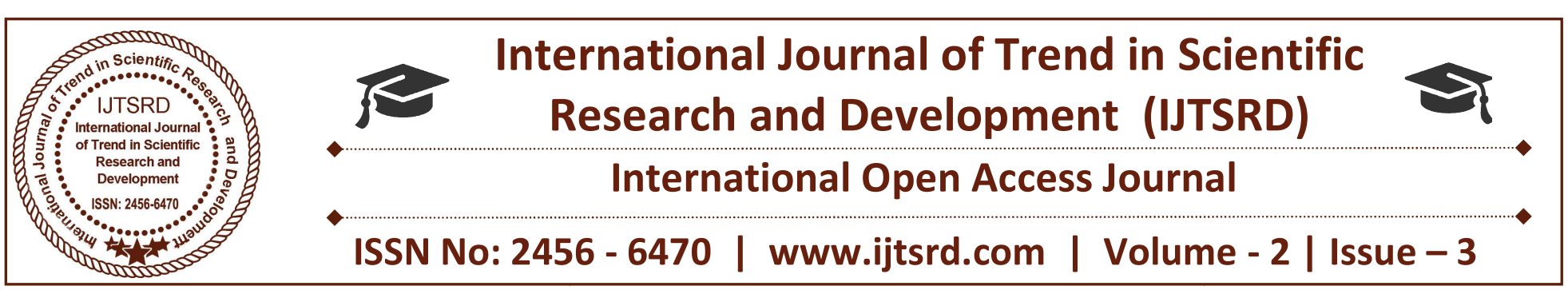

\title{
Role of Artificial Intelligence in Transforming Human Resource Management
}

\author{
Dr. Amol Murgai \\ Associate Professor, Department of M.B.A., International Centre for Excellence \\ in Engineering \& Management, Aurangabad, Maharashtra, India
}

\begin{abstract}
Authors like Isaac Asimov have for long imagined a future where machines that can govern themselves to help mankind. It was perhaps this imagination by the artists that led science on the path of inventing artificial intelligence in the first place. However, as things have advanced, science and AI have proven themselves to be much more than merely helpful for mankind so far. Machines have a long way to go before they reach the point envisioned by Asimov as they are still dependent on people to make moral judgment. Among the latest patrons of AI are the HR departments that have realized the wisdom and impact of assisted decision making about the most valuable resource of an organization - the people.

The present paper stresses upon the impact of Artificial Intelligence on Human resource management. The paper goes on discussing the areas of Human Resource wherein Artificial Intelligence proves to be useful.
\end{abstract}

Keywords: Human Resource, Talent Management, Computers

\section{Introduction to Artificial Intelligence}

Artificial intelligence (AI) has been the focus of thousands of technological developments for decades as mankind attempts to engineer increasingly sophisticated tools that can think, plan and adapt in ways that mimic the human brain. However, AI typically performs these processes at exponentially faster speeds than brains can manage without making computational errors or growing fatigued or bored. Artificial Intelligence (AI) is fast becoming a mainstream element of business. Artificial intelligence is transforming our lives at home and at work. In the workplace, artificial intelligence is evolving into an intelligent assistant to help us work smarter.

Artificial intelligence is playing a more significant role in business than ever before. Research firm IDC predicts the market for A.I. will grow from $\$ 8$ billion in 2016 to $\$ 47$ billion by 2020 , impacting all business practices across almost every industry. While the larger Asian economies like India and China have the privilege of humungous data, Singapore and Australia have a clear roadmap for leveraging their strong $R \& D$ capabilities for AI advancement. The applications of AI differ by demographics, ranging from financial services to logistics to healthcare and more recently, talent management. The wide presence of knowledgeintensive industries is compelling the government to explore automation and AI-led avenues to manage talent better. As a result, AI will have a profuse impact on human capital management, from an organizational perspective.

\section{Types of Artificial Intelligence}

Artificial intelligence (AI) is seen as the next wave of technological advancement with tools that can think, plan, and execute tasks that mimic human performance without stress or the need for a break from work. Artificial intelligence comes in narrow and strong levels, but most AI systems can be classified in three categories: voice recognition, bots and algorithms. There are actually many kinds of artificial intelligence that can range from calculating auto immune system performance to automating 
physical systems, but the three major areas that concern HR are voice recognition, robot automation and algorithms. Narrow-based AI functions are ideal for compiling data in HR systems such as verifying information, researching specific areas and solving other task-specific problems.

\section{Voice Recognition}

This type of AI can convert text into words and vice versa, search Internet sites, videos, podcasts and broadcasts for key phrases and subjects and deliver the information automatically to other analytic programs and on-demand in the preferred format of speech or text. An HR manager's AI personal assistants usually employ this technology. The primary function of voice recognition, however, is to trigger actions based on voice commands, such as opening a file, website or program, adjusting environmental controls, controlling household and office devices and other simple command functions.

\section{Bots}

The major search engines employ bots to search the Internet for keyword phrases. The usefulness of bots extends to learning, chatting, asking questions, giving directions, recalculating routes and other useful functions. Current AI systems can learn, but they have a long way to go before they can solve complex logic problems. Even the simplest decision involves thousands of variables. AI can accelerate finishing repetitive searching tasks exponentially.

\section{AI Algorithms}

AI algorithms are step-by-step instructions that guide AI functions. Sophisticated algorithms can be set to automate many HR functions such as gathering business intelligence, disseminating information to the right stakeholders, monitoring key performance benchmarks and tracking outside interests and social media activity of employees and recruitment prospects.

\section{Artificial Intelligence and Human Resource Management}

Artificial intelligence is not the future of the workplace; it is the present and happening today. HR leaders and experts are starting to realize the power and impact of data-driven statistics and insights when it comes to mellowing down risks and driving decision-making powers for organizational efficiency and people management.

With advanced data-driven technology such as artificial intelligence (AI) growing by leaps and bounds across businesses, it should not come as a surprise that HR experts are looking up to AI as their go-to or perfect pick-me-up tool for spot-on decision making and people management in future.

IBM and a number of startups are targeting intelligent assistants, also known as chatbots, or computer algorithms designed to simulate a human conversation, to recruit employees, answer HR questions, or personalize learning experiences. A survey of nearly 400 chief human resource officers conducted by the IBM Institute for Business Value found that half of the survey sample recognize the power of cognitive computing to transform key dimensions of HR, such as HR Operations, Talent Acquisition, and Talent Development.

HR leaders have started experimenting with all facets of AI to deliver value to their organizations. As intelligent assistants become more widely used in our personal lives, we will expect to see similar usage in the workplace.

For employees, chatbots deliver an unmatched level of employee experience, from real time answers for HR questions to personalized learning and development. In addition, they are critically important to the 3.7 million workers, or $2.8 \%$ of the workforce, who work remotely at least half time and do not have easy access to an HR department.

Talent acquisition and new hire on-boarding are ripe areas where intelligent assistants can tap multiple data sources to develop candidate profiles, schedule interviews, and make decisions about prospective job candidates.

Artificial intelligence in HR is likely to transform HR operations in three profound ways.

First is the emergence of the conversational interface, where we can talk to systems, ask questions and interact through chat. This can be supplemented by augmented and virtual reality, which is developing even faster than we thought. Second is machine learning, where software analyzes people-related data and offers smart recommendations and decisions. The third is the growth of predictive models, which are systems that can identify patterns and quickly find 
areas of risk, fraud and other possible performance problems.

\section{Few areas of implementing AI in Human Resource Management}

HR is one a discipline with extremely complex needs of data analytics and management. AI can assist in gathering large quantities of data and make predictive analysis in a matter of seconds, a task that would probably take months to do manually. This speed is crucial for global businesses as they compete in a highly fluid environment. The areas where AI can be useful in Human Resources are identified as discussed below -

1. Recruiting: There is a wide range of new AI tools used in recruiting. For example, there are AIbased chat systems that can communicate with candidates and quickly screen people. These systems already enable candidates to select the right job or shift, and they can dramatically reduce the time recruiters spend on candidate screening. This frees up recruiters to focus their energy on assessment and selling.

2. Learning: The skills and capabilities we need at work are constantly changing. We now have vendor software that can intelligently recommend videos or learning programs based on your job role, experience and peers. There are systems that automatically read documentation and create micro-learning programs, and even systems that read and interpret an employee's writing or activities to recommend learning options.

\section{Screening candidates}

Recruiters have to go through a vast number of candidate profiles and depend on manual processes, where they screen profiles and set up in-person discussions. An intelligent AI-enabled screening software can bring about much difference in this arena. By analyzing the necessary intricacies and past success factors in a particular job profile, the software can find the most suitable set of candidates with the required skills, experience and cultural fit for the organization. Industry giants like SAP, Facebook, IBM and Hilton Worldwide have added such analytics to their sourcing mechanisms and redefined the hiring experience completely.

\section{Interviewing candidates}

On an average, recruiters spend $30 \%$ of their time in scheduling and interviewing candidates. Reports reveal that more than $50 \%$ of the candidates don't hear back after submitting their resumes through traditional corporate pipelines. AI machines can figure out the right fit and engage potential candidates in an efficient manner. Intelligent machines that deliver custommade messages to potential candidates enable recruiters to focus on their time in swiftly closing offers.

\section{Leadership}

Today, there are vendors that can intelligently assess characteristics of high-performing leaders by sending messages to employees to assess a leader's capabilities. Leaders can then receive personalized coaching and can view dashboards comparing their management effectiveness against their peers.

6. Sourcing: There are a lot of new tools available to help $H R$ executives find candidates on the internet, a process known as sourcing. Sourcing is a manual, handcrafted process that companies undertake to find candidates for roles that are otherwise hard to fill, such as executives, salespeople and engineers. Now there is software that can search and intelligently match prospective candidates against the characteristics of a particular company and communicate with them to see if they're interested in the position.

\section{Employee Relations}

An AI-powered Chatbots can respond to the most common HR queries and it can schedule the meetings with you and your HR. Through HR analytics it can empower immediate managers to make better decisions through various information - Learning pattern, Performances Track records and Recognition Pattern.

\section{Benefits of using Artificial Intelligence in Human Resource Management}

The benefits of using AI in Human Resource Management have been identified in particular:

\section{Reducing Human Bias}

Artificial intelligence can help eliminate bias from certain performance metrics when needed. If an employee is simply not holding their own within a company, the data will show it and the AI can determine whether or not that employee should remain on the team. Similarly, $H R$ can use productivity measurements to determine manager effectiveness. 


\section{Increasing Efficiency and Insight in Candidate Assessment}

One of the key challenges for HR departments is selecting promising candidates from a large number of applicants. AI allows a number of the stages of the recruitment workflow to be automated. This means that more data can be gathered and assessed for each candidate and more candidates can be assessed overall. Innovative companies that provide AI recruitment solutions include Glider, who use sophisticated algorithms to assess candidates' skill sets. Recruitment agency I.T.S also makes use of AI to assess candidate suitability for job listings on behalf of their clients.

\section{Improving Relationships With Existing Employees}

HR departments are inundated with questions from employees. A significant number of these questions can be answered using AI. By programming automated email or instant messaging replies to common questions, the workload for HR departments can be reduced. This frees up HR personnel to answer more complex questions and engage more efficiently with a company's employees. Additionally, AI could soon play a role in other more mundane HR tasks. AI company x.ai recently launched "Amy," a virtual personal assistant that automates the process of scheduling meetings.

\section{Predictable turnover and attrition}

Human resource departments often deal with turnover and attrition after the employees have already left, but ai could predict such actions before they even take place. Using employee engagement data, whether it be from pulse surveys, brand advocacy or performance gamification, artificial intelligence could determine an employee's level of interest and give a prediction on whether they are trying to change positions. This would allow HR managers to account for potential job openings and preemptively hire new workers to more smoothly transition workflow.

\section{Recommended training methods}

Artificial intelligence can analyze the data from employee interactions and use that information to better tailor training sessions to the individual worker. Everyone learns in different ways and has varied skill sets, so allowing employees to learn to the best of their abilities will only improve on-boarding and productivity.

\section{Challenges around using AI in Human Resource Management}

- Though promising, Artificial Intelligence in HR can present a number of issues, the biggest being the quality of the data collected by companies.

- HR data is not managed effectively by most companies, and it is often in a number of different places. For instance, companies typically have five to seven different systems of record; therefore, applying an algorithm against any one data set can give you misleading results. It is a major challenge for companies to get all the people-related data into one place so it can be analyzed.

- The second challenge is policy. Explaining to employees how their data will be used and having policies to ensure that it's not misused remains a challenge. Most employees agree to their company having access to data when they join, and companies inform them that the data will be used for positive purposes. Sticking to those policies is key.

- The third is that there is no part of HR that's black and white. There's almost never 100\% agreement on who the best candidate for a job is or who should be promoted. Almost every decision in HR has some amount of judgment. So a lot of these algorithms may be helpful to eliminate that human judgment or bias, but that does not mean they are $100 \%$ correct.

\section{CONCLUSION}

Artificial intelligence is all about analysing, breaking down and transforming data into humanized format, which is easy to interpret and study. AI has steadily gained importance across all verticals of organizations like Marketing, HR, I.T, Sales to name a few. With the staggering influx of data in the human resource arena, artificial intelligence has started offering an ocean of insights in key areas that often go unnoticed such as productivity, managerial effectiveness, employee engagement and talent assessment to name a few. This has helped HR managers to understand their workforce in a much better way and foresee workforce trends as well as identify problem areas well in advance.

More importantly, AI also helps in solving one of the most critical challenges faced by HR people today i.e. creating and executing strategies for improvement, all by suggesting specific actions to tackle these problems well ahead of time. Since HR is a strategic 
business function, there is a vast scope in this vertical to adapt to newer technologies, for example, software applications like Human Capital Management have successfully embedded AI functionalities into its core processing engine and have proved to be a game changer. We do need to adapt ourselves to AI and the reason is simple - evolution. Technology is evolving and we need to stay abreast with it or risk losing our relevance to machines just as some artists had envisioned.

\section{REFERENCES}

1. $\mathrm{PwC}$, April http://www.pwc.com/CISAI?WT.mc_id=CT1PL52-DM2-TR1-LS4-ND6-BPA1-CN CIS-AIAIsocial
2. The Verge,

October

2016. https://www.theverge.com/2016/10/25/13381246/ otto-self-driving-truck-budweiser-first-shipmentuber

3. https://www.wired.com/2015/04/hire-like-google/

4. https://www.gapjumpers.me

5. https://textio.com

6. https://glider.ai

7. http://www.i-talentsolutions.co.uk

8. https://ideal.com

9. X.AI. https://x.ai

10. https://www.hirevue.com/products/onlinecoaching-software

2017. 11. https://www.butterfly.ai

12. https://www.nytimes.com/2016/10/17/technology/ ibm-is-counting-on-its-bet-on-watson-and-payingbig-money-for-it.html? 